\title{
Experimental and computational studies of novel coaxial 2D Bragg structures for a high-power FEM
}

\author{
I.V. Konoplev ${ }^{\mathrm{a}, *}$, A.D.R. Phelps ${ }^{\mathrm{a}}$, A.W. Cross ${ }^{\mathrm{a}}$, K. Ronald ${ }^{\mathrm{a}}$, P. McGrane ${ }^{\mathrm{a}}$, \\ W. He ${ }^{a}$, C.G. Whyte ${ }^{a}$, N.S. Ginzburg ${ }^{b}$, N.Yu. Peskov ${ }^{b}$, A.S. Sergeev ${ }^{b}$, M Thumm $^{\mathrm{c}}$ \\ ${ }^{a}$ Department of Physics, University of Strathclyde, Glasgow, G4 ONG, UK \\ Institute of Applied Physics, RAS, Nizhny Novgorod 603950, Russia \\ ${ }^{\mathrm{C}}$ Universät Karlsruhe, Instü̈ntHochfrequenz technik und Electronik, D-76021 Karlsruhe, Germany
}

\begin{abstract}
Two-dimensional (2D) coaxial Bragg structures have been suggested for use in high-power Free Electron Masers (FEM) to synchronize radiation from different parts of an oversized annular electron beam. In this paper, the simulations of field evolution using the three-dimensional code MAGIC are carried out and results are presented. An investigation of 2D Bragg structures obtained by corrugating the inner surface of the outer conductor of a coaxial waveguide or by lining the surface of a smooth waveguide with a dielectric material, which has a bi-periodic permittivity, has been conducted. Experimental studies of 2D Bragg structures were also undertaken and the good agreement between experimental measurements and theoretical predictions is demonstrated. Measurements of a $7 \mathrm{~cm}$ diameter annular electron beam produced by a high-current accelerator to be used to drive the FEM are presented and the experimental set-up discussed.
\end{abstract}

Keywords: FEM; 2D feedback; Microwaves; Annular e-beam

\section{Introduction}

Two-dimensional (2D) coaxial Bragg structures have recently been under intensive theoretical [1-5] and experimental [6-8] study. These structures have been proposed for application in high-power microwave electronics [1] to synchronize the radiation from different parts of an oversized electron beam which can be used to drive a highpower Free Electron Maser (FEM). An example of such a structure with periodic perturbation on the surface of the inner conductor is shown in Fig. 1. The studies of novel 2D Bragg structures for a high-power FEM driven by an annular electron beam have been conducted and results are presented. We also present measurements of the annular electron beam produced by a high-current accelerator (HCA), which will be used to power 


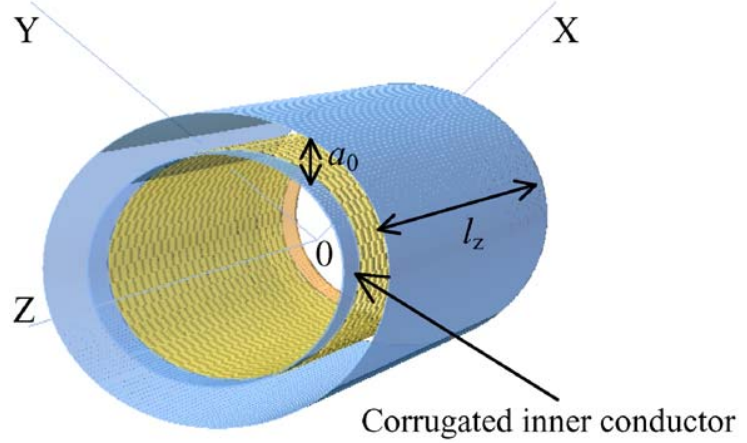

Fig. 1. The schematic diagram of the coaxial 2D Bragg structure with corrugated inner conductor.

the FEM experiment. In Section 2 of the paper the basic model is discussed, results of numerical simulations are presented and compared with experimental data. In Section 3, the field evolution inside the single section 2D Bragg cavity is considered. Experimental measurements of the annular electron beam, to be used to drive the high-power FEM are presented in Section 4. From these measurements the operating parameters of the FEM experiment are estimated in the Conclusion.

\section{Basic model}

The 2D Bragg corrugation of the waveguide on either inner or outer conductor surfaces can be presented as

$r(z, \varphi)=R_{\text {in,out }}+a_{1} \cos \left(\bar{k}_{z} z\right) \cos (\bar{m} \varphi)$

where $R_{\text {in,out }}$ are the radii of inner (in) and outer (out) conductors, $a_{1}$ is the amplitude of the corrugation, $\bar{k}_{z}=2 \pi / d_{z}$ and $d_{z}$ is the period of the corrugation along the longitudinal coordinate $z$ and $\bar{m}$ is the corrugation variation number along the azimuthal coordinate $\varphi$. In the Bragg structures studied, the amplitude of the perturbations $a_{1}$ is small in comparison with the operating wavelength $\lambda_{0}$ and the distance between the inner and outer conductors. The dispersion equation for the eigenwaves of the coaxial waveguide takes the following form [9]:

$k^{2}=\omega^{2} / c^{2}=k_{z}^{2}+k_{\perp}^{2}$

where $\omega$ is the wave frequency, $k_{z}$ and $k_{\perp}$ are the longitudinal and transverse wavenumbers, respectively. The RF field inside the 2D Bragg structure can be presented as a superposition of four waves: $\mathrm{A}_{ \pm}$propagating in $\pm z$ direction and $\mathrm{B}_{ \pm}$are near cut-off waves as shown in the schematic diagram of Fig. 2a with the structure's eigenvectors represented as $\vec{k}_{ \pm}$. The $2 \mathrm{D}$ periodic corrugation provides a wave scattering such that counterpropagating waves are coupled indirectly. The partial wave $\mathrm{A}_{+}$propagating in the $+z$ direction is scattered into near cut-off waves $\mathrm{B}_{+}$, which scatter into waves $\mathrm{A}_{+}$. This ensures that the following loop $\mathrm{A}_{+} \leftrightarrow \mathrm{B}_{ \pm} \leftrightarrow \mathrm{A}_{-}$is completed and allows the formation of a $2 \mathrm{D}$ feedback circle. Taking into account the case when the partial wave $\mathrm{A}_{+}$is represented by a TEM wave, one finds that to obtain an efficient coupling of the waves $\mathrm{A}_{+}$ $\leftrightarrow \mathrm{B}_{+}$the following resonance conditions should be satisfied:

$k_{z}=k_{z}^{\prime} \cong \bar{k}_{z},|\bar{m}|=|M|$ and $|k| \approx\left|k_{\perp}^{\prime}\right|=\left|k_{\perp}\right|$

where $M$ is the azimuthal wavenumber and $k_{\perp}, k_{\perp}^{\prime}$ are the full transverse wavenumbers of the partial waves $\mathrm{B}_{ \pm}$and $k$ is the full wavenumber of the incident wave $\mathrm{A}_{+}$.

Using the three-dimensional PIC code MAGIC, the field scattering on the 2D Bragg corrugation has been investigated. The snapshot at the crosssection corresponding to the centre of the structure

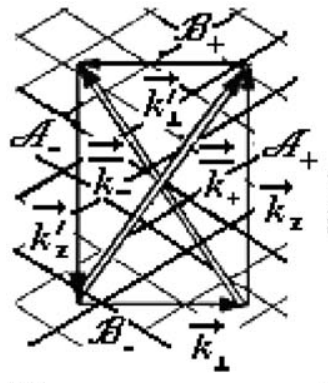

(a)

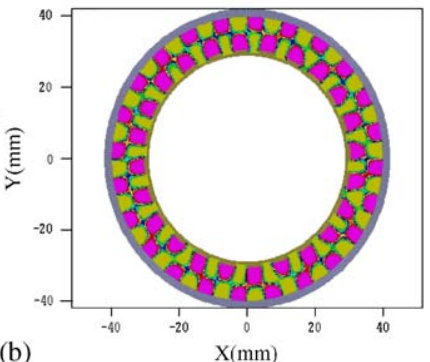

(b)
Fig. 2. (a) The schematic diagram of the 2D feedback circle; and (b) the snapshot of the longitudinal component of the magnetic field inside the 2D Bragg structure. 
shows the profiles of the waves $\mathrm{B}_{ \pm}$(Fig. 2b) due to scattering of the launched TEM wave of the coaxial waveguide. The TEM wave has no field variation along the radial and azimuthal coordinate, also the field component $\left(H_{z}\right)$ observed in simulations (Fig. 2b) does not exist in the field of either incident or reflected TEM waves $\left(\mathrm{A}_{ \pm}\right)$and can only be attributed to the near cut-off wave $\mathrm{TE}_{24,1}$ of the coaxial waveguide (partial waves $\left.\mathrm{B}_{ \pm}\right)$. The change of the polarity along the azimuthal and radial coordinates is obvious and $(24,1)$ variations are clearly evident. This agrees well with conditions (3) and the waveguide dispersion relation. Considering dispersion relation (2) and condition (3), one notes that to excite a TE mode with zero radial variation for the same waveguide parameters and $\bar{k}_{z}$ the azimuthal variation index of the corrugation should be $\bar{m}=28$ (Fig. 3).

In the recent studies $[7,8]$, the existence of $2 \mathrm{D}$ scattering has been experimentally demonstrated and shown that the 2D Bragg structure can operate as a narrow frequency band reflector. Thus in Fig. 4, the transmission coefficient from the 2D Bragg structure with a corrugated inner conductor obtained using analytical theory developed in Refs. [4-8] is presented (bold line) and compared with experimental result (thin line) obtained using a Scalar Network Analyser (Fig. 4a). The results are also compared against data obtained from numerical simulations conducted using the code MAGIC (Fig. 4b) and the good agreement between the results obtained using the different methods is clearly evident.

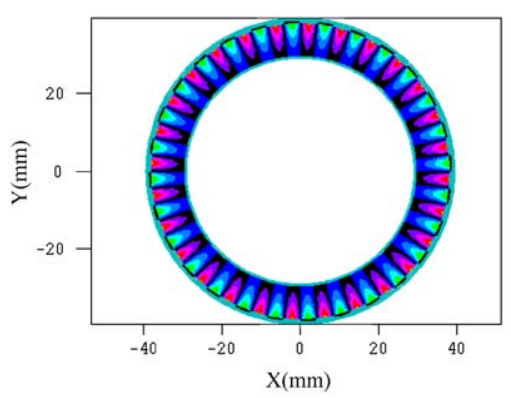

Fig. 3. The snapshot of the longitudinal component of the magnetic field inside the 2D Bragg structure.
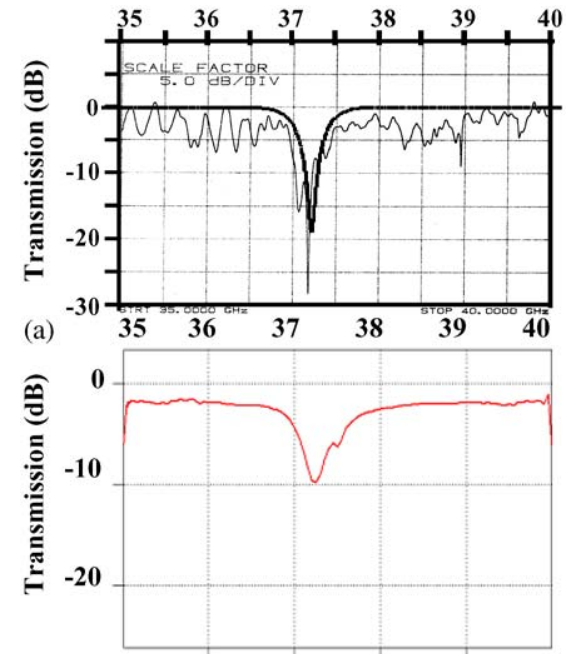

(b)

Frequency $(\mathrm{GHz})$

Fig. 4. The transmission coefficient from the 2D Bragg structure obtained (a) from analytical formula [4-8] (thick line) and experimental data (thin line); and (b) using the PIC code MAGIC.

\section{Computational study of RF field evolution}

Let us consider the 2D Bragg structure as a part of the interaction space of an FEM. The selective properties of the $2 \mathrm{D}$ Bragg structure as a single section cavity has been recently under intensive investigation $[1-5,10]$. It has been demonstrated that after some period of time only the fundamental mode with the highest $Q$-factor having one variation along the longitudinal coordinate can be found in the cavity. This has also been confirmed for the cavity based on a 2D Bragg structure considered in this paper. Analysing the time decay of the field inside the cavity excited by RF pulses [10] formed by TEM and $\mathrm{TE}_{1,0}$ waves, respectively, it has been found that the time decay of the "nonsymmetric" mode is $\tau \approx 10 \mathrm{~ns}$ which corresponds to $Q \approx 1100$ while the time decay of the "symmetric" mode is $\tau \approx 15 \mathrm{~ns}(Q \approx 1600)$. This may result in effective mode selection over the wave's azimuthal index allowing single mode operation of an FEM driven by an oversized beam. One notes that the mode's $Q$-factor tends to decrease with increase in the azimuthal index $[2-5,7]$.

Considering a two-mirror cavity based on the 2D Bragg structures, an effective mode selection 
over the wave azimuthal index is also expected. One of the ways to provide the mode selection is to ensure that spurious azimuthally non-symmetric modes (for instance $\mathrm{TE}_{1,0}$ ) excited inside the cavity lie outside the reflection band of either the input or output mirrors. This can be achieved if the input and output 2D Bragg mirrors have different azimuthal variation indices, i.e. $\bar{m}_{1} \neq \bar{m}_{2}$. In the high-power Strathclyde FEM experiment, the use of input and output mirrors with $\bar{m}_{1}=24, \bar{m}_{2}=$ 28 , respectively, is under consideration. Comparing Fig. $4 \mathrm{~b}$ where $\bar{m}_{1}=24$ and Fig. 5 where $\bar{m}_{1}=$ 28 , it is clear that the reflection bands for the azimuthally symmetric wave are located in the same frequency band. Assuming now that a spurious $\mathrm{TE}_{1,0}$ wave is excited inside the cavity, one finds that the cut-off frequencies of the partial waves coupled on the corrugation and which define the location of the reflection zones, are different for input and output reflectors. This results in frequency shift of the reflection zones' centres and therefore to avoid any possibility of the excitation of such a mode, the overlap of reflection zones for the input and output reflectors should be avoided. This can be achieved by varying the coupling coefficient, whose value affects the effective width of the reflection zone [5-8]. In Fig. 3, the snapshot of the profile of the waves $\mathrm{B}_{ \pm}\left(H_{z}\right)$ is shown at a cross-section, in the centre of the structure. Taking into account the field structure of the $\mathrm{TE}_{28,0}$ mode of a coaxial waveguide, one notes that to obtain an effective wave coupling the corrugation has to be machined on the surface of the outer conductor. We are also

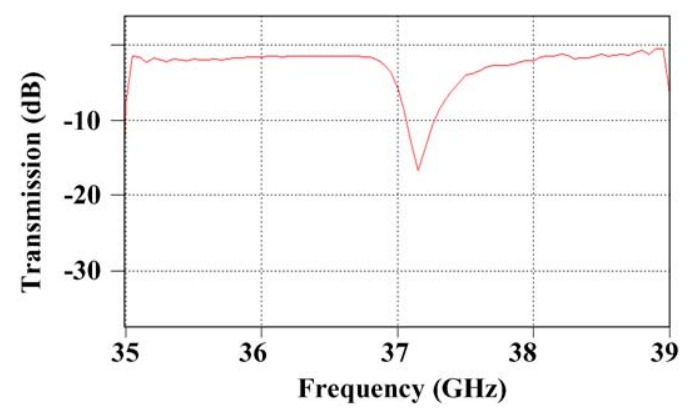

Fig. 5. The transmission coefficient from the 2D Bragg structure obtained using the PIC code MAGIC. considering using a 2D Bragg structure which can be achieved by lining the surface of the outer conductor with a bi-periodic dielectric which is beneficial if a pulsed guide solenoid is used to confine the electron beam. Using the PIC code MAGIC field scattering on the outer conductor surface lined by a bi-periodic dielectric has been simulated and results similar to those presented in Fig. 4 have been obtained.

\section{Annular electron beam formation}

The HCA which is to be used to drive the FEM, is based on a magnetically insulated explosive emission carbon cathode able to produce a thin annular electron beam. In Fig. 6a, the photograph of the experiment is presented and the following components are indicated: (a) pulsed wiggler and capacitor bank power supply; (b) table containing ten $250 \mu \mathrm{F}, 20 \mathrm{kV}$ capacitors used to generate up to $0.8 \mathrm{~T}$ from a large diameter $(30 \mathrm{~cm})$ pulsed solenoid of length $2.5 \mathrm{~m}$; (c) X-ray shielded enclosure; (d) coaxial cavity, electron beam diagnostics and guide solenoid; (e) ignitron switches with solid-state trigger units; (f) diode tank containing electron gun and transmission line output spark gap. In Fig. 6b, a schematic diagram of the HCA shows: (I) the Marx pulsed power

(a)
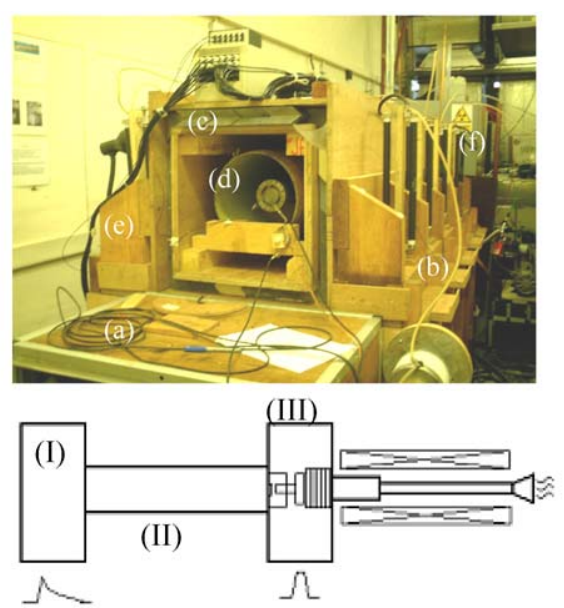

Fig. 6. (a) Photographs of the experimental set-up; and (b) the schematic diagram of the experimental set-up. 
supply (a 15 stage, 1.5 MV Marx bank generator) connected via a transmission line (II) to the plasma flare emission electron gun (III). The power supply allows the intermediate electrode of the transmission line to be charged up to $1 \mathrm{MV}$ and generates an output pulse of duration $\sim 300 \mathrm{~ns}$. The transmission line of length $2 \mathrm{~m}$ is a coaxial line which has an outer conductor diameter of $1 \mathrm{~m}$, filled with high-purity de-ionized water $(18 \mathrm{M} \Omega$ / $\mathrm{cm}$ ), an impedance of $4.7 \Omega$ and a total capacitance of $25 \mathrm{nF}$. The transmission line is switched into the load using a high-pressure (10 bar) nitrogen-filled spark-gap. At the input of the load a rectangular voltage pulse with a flat top of length up to $200 \mathrm{~ns}$ is formed. The high-voltage accelerator was connected to the electron gun and triggered to coincide with the firing of the pulsed solenoid. A high-current (1.6 kA), high-voltage (up to $500 \mathrm{kV}$ ) large-diameter (mean diameter is $7 \mathrm{~cm}$ ) annular electron beam was generated. In Fig. 7a, a witness plate beam diagnostic positioned at the input to the coaxial interaction region, $15 \mathrm{~cm}$ from the
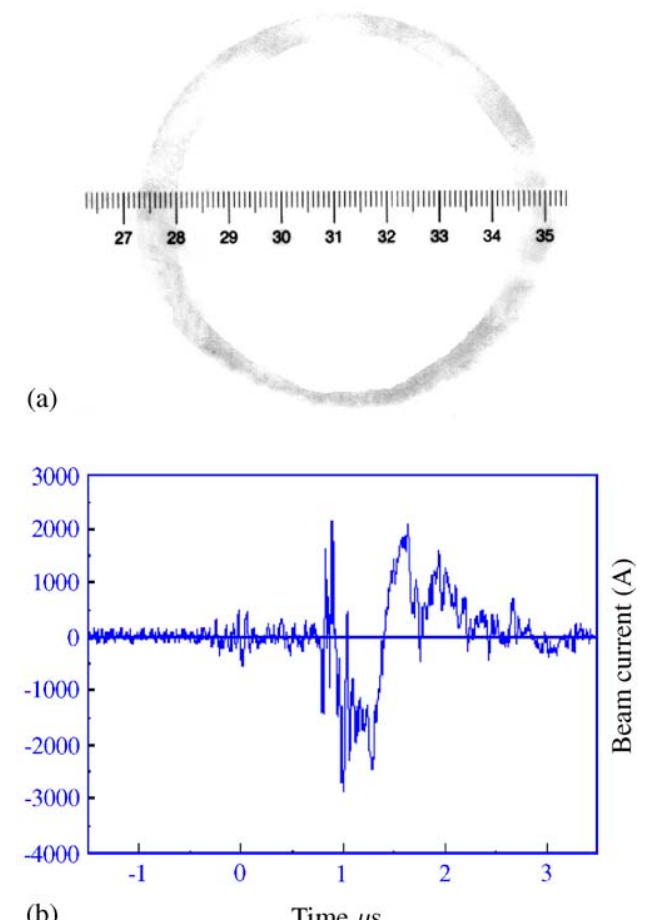

(b)

Time $\mu \mathrm{s}$

Fig. 7. (a) Photograph of the electron beam trace; and (b) the electron beam current measured using a Rogowski coil. anode plate, was used to obtain the transverse position of the electron beam, with a beam current of $1.6 \mathrm{kA}$ measured (Fig. 7b) using an in-line Rogowski coil beam diagnostic.

\section{Conclusion}

We have discussed the basic model and field scattering on the 2D Bragg corrugation. The formation of $2 \mathrm{D}$ feedback loop has been investigated. Good agreement between simulations and basic model predictions is demonstrated. The results of simulations have also demonstrated the possibility to obtain efficient azimuthal mode selection in an oversized cavity based on 2D Bragg structures. Taking into account the experimental measurements obtained from the HCA, the parameters of the FEM experiment may be estimated. An electron beam of power $750 \mathrm{MW}$ has been measured. Assuming an FEM output efficiency of $15 \%$ which has been predicted in Refs. [1-7], the generation of $\sim 100 \mathrm{MW}$ of power at the operating frequency of $37.5 \mathrm{GHz}$ is predicted.

\section{Acknowledgements}

The authors would like to thank QinetiQ, EPSRC, INTAS and the University of Strathclyde for support of this work.

\section{References}

[1] N.S. Ginzburg, et al., Opt. Commun. 96 (1993) 254.

[2] N.S. Ginzburg, et al., Tech. Phys. 41 (5) (1996) 465.

[3] N.S. Ginzburg, et al., IEEE Trans. Plasma Sci. 24 (1996) 770.

[4] I.V. Konoplev, et al., Nucl. Instr. and Meth. A 445 (2000) 236.

[5] N.S. Ginzburg, et al., J. Appl. Phys. 92 (2002) 1619.

[6] I.V. Konoplev, et al., Proceedings of IEE Symposium on Pulsed Power 2000, 2000, p. 412.

[7] A.W. Cross, et al., Nucl. Instr. and Meth. A 475 (2001) 164.

[8] A.W. Cross, et al., Appl. Phys. Lett. 80 (2002) 1517.

[9] A.F. Harvey, Microwave Engineering, Academic Press, London, 1963.

[10] N.S. Ginzburg, et al., Technical Physics, 48 (12) (2003) 54. 